University of New Hampshire

University of New Hampshire Scholars' Repository

$11-1-2012$

\title{
Strength asymmetry increases gait asymmetry and variability in older women.
}

\author{
Dain P. LaRoche \\ University of New Hampshire, Durham, dain.laroche@unh.edu \\ Summer B. Cook \\ University of New Hampshire, Durham \\ Krzysztof Mackala \\ University School of Physical Education
}

Follow this and additional works at: https://scholars.unh.edu/kinesiology_facpub

\section{Comments}

The publisher's final edited version of this article is available at: https://dx.doi.org/10.1249/MSS.0b013e31825e1d31

\section{Recommended Citation}

D.P. LaRoche, Cook, S.B., Mackala, K. (2012) Strength asymmetry increases gait asymmetry and variability in older women. Medicine \& Science in Sports \& Exercise. 44(11):2172-2181, 2012.

This Article is brought to you for free and open access by the Kinesiology at University of New Hampshire Scholars' Repository. It has been accepted for inclusion in Kinesiology Scholarship by an authorized administrator of University of New Hampshire Scholars' Repository. For more information, please contact Scholarly.Communication@unh.edu. 


\title{
Strength Asymmetry Increases Gait Asymmetry and Variability in Older Women
}

\author{
Dain P. LaRoche ${ }^{1}$, Summer B. Cook ${ }^{1}$, and Krzysztof Mackala ${ }^{2}$ \\ Summer B. Cook: Summer.Cook@unh.edu; Krzysztof Mackala: krzysztof.mackala@awf.wroc.pl \\ ${ }^{1}$ Department of Kinesiology, University of New Hampshire, 124 Main St. Durham, NH 03824, \\ Phone: (603) 862-4859 \\ ${ }^{2}$ Department of Track and Field, University School of Physical Education, Ul. Paderewskiego 35, \\ 51-612 Wrocław, Poland, Phone: +48 713473147
}

\begin{abstract}
Purpose-The aim of the research was to determine how knee extensor strength asymmetry influences gait asymmetry and variability since these gait parameters have been related to mobility and falls in older adults.

Methods-Strength of the knee extensors was measured in 24 older women $(65-80 \mathrm{yr})$. Subjects were separated into symmetrical strength $(\mathrm{SS}, \mathrm{n}=13)$ and asymmetrical strength $(\mathrm{SA}, \mathrm{n}$ $=11$ ) groups using an asymmetry cutoff of $20 \%$. Subjects walked at a standard speed of $0.8 \mathrm{~m} \mathrm{~s}^{-1}$ and at maximal speed on an instrumented treadmill while kinetic and spatiotemporal gait variables were measured. Gait and strength asymmetry were calculated as the percent difference between legs and gait variability as the coefficient of variation over twenty sequential steps.
\end{abstract}

Results-SA had greater strength asymmetry $(27.4 \pm 5.5 \%)$ than SS $(11.7 \pm 5.4 \%, \mathrm{P}<0.001)$. Averaged across speeds, SA had greater single (7.1\% vs. $2.5 \%)$ and double-limb support time asymmetry ( 7.0 vs. $4.3 \%)$ than SS and greater single-limb support time variability $(9.7 \%$ vs. $6.6 \%$, all $\mathrm{P}<0.05)$. Group $\times$ speed interactions occurred for weight acceptance force variability $(\mathrm{P}=$ $0.02)$ and weight acceptance force asymmetry $(\mathrm{P}=0.017)$ with greater variability at the maximal speed in SA $(5.0 \pm 2.4 \%$ vs. $3.7 \pm 1.2 \%)$ and greater asymmetry at the maximal speed in SA (6.4 \pm $5.3 \%$ vs. $2.5 \pm 2.3 \%)$.

Conclusion-Gait variability and asymmetry are greater in older women with strength asymmetry and increase when they walk near their maximal capacities. The maintenance of strength symmetry, or development of symmetry through unilateral exercise, may be beneficial in reducing gait asymmetry, gait variability, and fall risk in older adults.

\section{Keywords}

Elderly; Mobility Limitation; Walking; Quadriceps Muscle; Aging

Copyright (C) 2012 American College of Sports Medicine

Address for correspondence and reprints: Dain P. LaRoche, Ph. D. Department of Kinesiology, 124 Main St. Durham, NH 03824, Phone: (603) 862-4859, Fax: (603) 862-0154, Dain.LaRoche@unh.edu.

The authors report no conflicts of interest.

The results of the present study do not constitute endorsement by the American College of Sports Medicine.

Publisher's Disclaimer: This is a PDF file of an unedited manuscript that has been accepted for publication. As a service to our customers we are providing this early version of the manuscript. The manuscript will undergo copyediting, typesetting, and review of the resulting proof before it is published in its final citable form. Please note that during the production process errors may be discovered which could affect the content, and all legal disclaimers that apply to the journal pertain. 


\section{Introduction}

Increased fall risk and declining mobility threaten the health and independence of older adults. Falling is a multifactorial problem linked to muscle weakness, gait and balance deficits, visual defects, arthritis, depression, and reduced cognitive function (1). Strength asymmetry $(27,33)$, gait asymmetry $(4,42)$, and gait variability $(5,14)$ have each been identified as independent factors related to falling in older adults. Despite the potential connection between these measures, there is a lack of research that has investigated whether increased strength asymmetry leads to greater gait asymmetry and variability in older adults. Strength asymmetry, or the percent difference in strength for a specific muscle group from one side of the body to the other, may potentially be due to limb dominance (20) or unilateral pathology (35) and has been shown to increase with age (27). Yet, strength asymmetries do not necessarily follow limb dominance, and laterality of strength may vary for each joint action or functional task (38).

While estimates of strength asymmetry in young adults are between $5-15 \%(20,27)$, older adults exhibit asymmetries of approximately $15-20 \%$ (27, 33). For example, Carabello and colleagues demonstrated that older adults had greater knee extensor strength asymmetry $(\approx 15 \%)$ than middle-aged adults $(10 \%)$. When they compared mobility limited older adults to those with normal mobility, they showed greater knee extensor power asymmetry in mobility limited individuals ( $21 \%$ vs. $12 \%$ ). Furthermore, strength and power asymmetry appear to be higher in elderly fallers than in nonfallers $(27,33)$. In fact, Skelton et al. showed that $60 \%$ of fallers had knee extensor power asymmetry greater than $10 \%$, while only $13 \%$ of nonfallers demonstrated this level of power asymmetry (33).

Gait asymmetry, or differences in the bilateral behavior of the legs during walking, is thought to arise from limb dominance, disease, leg length discrepancies, and strength imbalances (30). For example, step length (i.e. the distance from contact of one foot to contact of the opposite foot) could differ between legs as a consequence of strength asymmetry. Gait asymmetry has been shown to increase with age, is higher in elderly fallers than nonfallers, and is inversely correlated with preferred gait speed (42). In older adults, Bautmans et al. showed that asymmetrical gait was positively correlated to fall risk and dependency in activities of daily living that encompassed bathing, dressing, toilet, transfer, continence, and feeding activities (4). In addition to negatively affecting physical function, gait asymmetry may reduce the rhythmicity of walking resulting in increased gait variability.

Gait variability, which has routinely been measured as the spatial or temporal inconsistency of strides (e.g. time from contact of one foot to contact of the same foot), is also strongly related to falling. Hausdorff et al. (14) demonstrated in a cross-sectional study that the coefficient of variation (CV) for stride time, a measure of gait variability, was higher in elderly fallers $(5.3 \%)$ than in elderly nonfallers $(1.7 \%)$ and young $(1.1 \%)$. They also showed during a one year prospective study that high stride time variability at baseline increased the risk for falling during follow up by fivefold. These researchers established that stride time variability was related to health (Charlson Comorbidity Index), performance on the Mini-Mental State Examination, independence in activities of daily living, health-related quality of life, age, static balance, preferred gait speed, knee and grip strength (15).

Similarly, Brach et al. demonstrated in subjects who had self-selected gait speeds above 1.0 $\mathrm{m} \mathrm{s}^{-1}$ that stride width CV was greater in those with a history of falls $(27 \%)$ than in those who had not fallen in the previous year (20\%), yet no differences in CV were observed in those who walked slower than $1.0 \mathrm{~m} \mathrm{~s}^{-1}$ (5). In opposition to these studies, Paterson et al. did not show a difference for spatiotemporal gait variability between fallers and nonfallers, but did show greater right-left asymmetry in fallers for a stride time derived fractal scaling index (26). 
While walking, the muscles of the lower-extremity play important roles in body support, propulsion and stability and it is therefore reasonable to speculate that relationships may exist between strength asymmetry, gait asymmetry, and gait variability. If as the result of a strength imbalance, the forces generated by the lower-extremity during walking are applied unequally between legs (31), gait parameters like step length, step time, and single-limb support time would be expected to be different from side-to-side and step-to-step. Consequently, strength asymmetry in the lower-extremities of older adults may negatively affect the quality of movement resulting in increased gait asymmetry and variability. This concept may help to explain the relationship between low strength and increased gait variability identified by Hausdorff et al. (15) and by Kang and Dingwell (18). If relationships between strength asymmetry, gait asymmetry, and gait variability can be identified, restoration of strength symmetry through focused resistance training programs could aid in the reduction of fall risk and maintenance of mobility.

The purpose of this study was to investigate the relationship between lower extremity strength asymmetry, gait asymmetry, and gait variability in older women. A second purpose was to associate the spatial and temporal measures of variability to kinetic gait parameters. We hypothesized that older women with knee extensor strength asymmetry greater than $20 \%$ will exhibit increased asymmetry and variability of spatial, temporal, and kinetic gait variables. We also hypothesized that spatial and temporal gait variability measures would be correlated to kinetic gait variability measures.

\section{Methods}

\section{Participants}

Twenty-four women between the ages of 65-80 $\mathrm{yr}$ who were able to walk without assistance, lived independently, and had no major cardiovascular, muscular, skeletal or neurological conditions participated in the study (Table 1). The women were separated into symmetrical strength (SS, $n=13)$ and asymmetrical strength $(\mathrm{SA})$ groups $(\mathrm{n}=11)$ based on a knee extensor strength asymmetry of $20 \%$. Asymmetry was calculated according to equation 1 as was done previously by Carabello et al. (7).

$$
\text { Asymmetry }=\frac{\mid \text { Weak leg value }- \text { Strong leg value } \mid}{\text { Strong leg value }} \times 100 \%
$$

Women whose weak-strong leg knee extensor strength asymmetry was greater than or equal to $20 \%$ were classified as asymmetrical and those whose strength asymmetry was less than $20 \%$ were classified as symmetrical. While healthy, younger adults exhibit leg strength asymmetries of 5 - 15\% (20,27), the work of Perry et al (27) and Skelton et al. (33) suggest that older adults exhibit average leg strength asymmetries closer to $15-20 \%$. These studies provide the basis for the $20 \%$ criterion used in the current study. Prior to participation, subjects had to provide written consent from their primary care providers and gave their own written, informed consent to participate in the research. The research protocol was approved for the use of human subjects by the university's Institutional Review Board.

\section{Procedures}

The study required two visits to the laboratory separated by two to seven days. During the first visit, height and mass were measured and subjects' habitual, overground gait speed was assessed using a timed four-meter walk. Next, participants were familiarized to both the knee extensor strength and treadmill walking protocols. During the second visit, maximal torque and maximal rate of torque development (RTD) of the knee extensors were determined for each leg and subjects were classified into the SS and SA groups. After ten 
minutes of rest, the participants' gait was assessed during treadmill walking at both standard and self-selected maximal speeds.

\section{Strength and Rate of Torque Development Measurement}

Measurements of maximal knee extensor torque and RTD were conducted using computerized dynamometry in a seated position with a hip angle of $100 \mathrm{deg}$ (HUMAC Norm, CSMI, Stoughton, MA, USA). The subjects' torsos and legs were secured to the dynamometer using nylon straps to restrict movement at the hip and isolate the knee extensors. They then performed two maximal voluntary isometric knee extensions at a knee angle of $105 \mathrm{deg}$, contracting for two seconds, for each leg. The contractions were initiated by a visual cue and participants were instructed to produce as much force as quickly as possible exerting maximal effort over the two-second contraction period. Thirty seconds of recovery was provided between trials and the order of testing was randomized between the right and left legs. The analog torque output of the dynamometer was sampled using a data acquisition system (BIOPAC MP100, Biopac Systems, Inc., Goleta, California, USA) at 1 $\mathrm{kHz}$ and smoothed using the mean torque calculated over overlapping 50 sample segments. Using the data acquisition system's software (BIOPAC AcqKnowledge, Biopac Systems, Inc., Goleta, California, USA), maximal torque was determined as the highest torque produced over the two trials and was recorded for each leg. This software was also used to determine the maximal, instantaneous rate of torque development, which was calculated as the slope of the torque vs. time curve in overlapping 50 sample segments.

\section{Gait assessment}

The participants walked on a motorized, instrumented treadmill (Gaitway II, Kistler Instrument Corp., Amherst, NY, USA) that recorded spatial, temporal and kinetic gait variables for each foot over twenty, sequential steps (ten strides). A minimum of three $20 \mathrm{~s}$ data collections were recorded and the first successful trial that captured every foot strike was used for analysis. Participants walked at both a standard speed of $0.83 \mathrm{~m} \mathrm{~s}^{-1}$ and at a self-selected, maximal speed for approximately two minutes each. For the maximal speed trial, they were specifically instructed to "walk as fast as you comfortably can as if you are late for an important event". The analog force and center of pressure (COP) data from the treadmill's force plates were sampled at $100 \mathrm{~Hz}$ by the treadmill's analog-digital board (PCIM-DAS1602/16, Measurement Computing, Norton, Massachusetts, USA), using a personal computer and software (Gaitway v. 2.0.8.50, Kistler Instrument Corp., Amherst, NY, USA) and were recorded independently for both right and left steps. For each step, the Gaitway software performed an automated analysis that identified the peak vertical ground reaction force for both the first (weight acceptance) and second (push-off) force peaks. The weight acceptance rate was then calculated as the slope of the force vs. time curve between $10-90 \%$ of the peak weight acceptance force and the push-off rate was calculated as the slope of the force vs. time curve between $90-10 \%$ of the push-off peak force.

Using the duration of each foot's force measurements, contact time with the treadmill deck was determined and used by the Gaitway software to calculate step time, single-limb support time and double-limb support time. Step time was determined as the duration from the initial force measurement of one foot to the initial force measurement of the opposite foot. Singlelimb support time was calculated as the duration for which force was recorded for only one foot and double-limb support time was the duration of the overlap of the right and left foot force data. The software also used COP data to determine spatial gait parameters. Step length was measured as the distance from the initial COPx (anteroposterior) at foot contact to the initial COPx of the contralateral foot at contact. Because treadmill walking is stationary with respect to the ground, the Gaitway system uses treadmill belt speed to determine the distance traveled in a given time to assign increasingly distant COPx with 
each step. Stride width was calculated as the average distance between the right and left foot COPy (mediolateral). Foot strike location was determined as the average COPy during stance of the strong leg and provided a measure of the mediolateral location of each step on the treadmill deck. For analysis, gait measurements (other than foot strike location and stride width) were recorded for both the right and left legs over the twenty sequential steps. Foot strike location was recorded for the strong leg only and stride width was recorded for each of the ten strides.

\section{Data Processing}

Trial data were exported from the Gaitway software to a spreadsheet program where for each subject and each speed the mean scores of the gait variables were determined across all steps (right and left together) as well as for each foot. For the determination of gait variability, the standard deviation of each variable was computed across all the steps in a trial (right and left together) at both standard and maximal speeds. The asymmetries of spatial, temporal and kinetic gait variables were calculated with the same formula used to determine strength asymmetry (eq. 1.) To determine gait asymmetry the mean score of the ten steps in a trial, for the strong and weak legs, was used for each variable and entered into equation 1 . To provide a unitless measure of gait variability within a twenty-step trial (included both right and left legs), coefficients of variation (CV) were determined for each gait measure (with the exception of foot strike location) according to equation 2 as performed previously by Hausdorff et al. (14).

$$
\text { Gait Variability }(\mathrm{CV})=\frac{\text { Standard deviation of steps in a trial }}{\text { Mean of steps in trial }} \times 100 \%
$$

The standard deviation of foot strike location was used instead of CV because the average position of the foot strike is irrelevant and using it in the calculation of CV would have biased the variability measure depending on the subject's mediolateral position on the treadmill.

\section{Statistical Analysis}

All statistical tests were performed using a statistical software package (IBM SPSS Statistics v. 19, Armonk, New York, USA) and all data are presented as mean \pm standard deviation. The normality of the data were confirmed with boxplots and a test of homogeneity of variances using the Levene statistic. To determine the consistency of the intralimb measurement of maximal knee extensor torque between trials 1 and 2, the intraclass correlation coefficient was calculated using Chronbach's alpha. Then the standard error of the measurement (SEM) of maximal knee extensor torque was calculated. For SA, a paired T-test was used to determine if the average bilateral strength asymmetry exceeded the average difference in intralimb knee extensor torque observed between trials 1 and 2 .

Differences between groups for subject descriptive variables were assessed using independent T-tests. A $2 \times 2$ (group $\times$ speed), repeated measures analysis of variance was used to compare basic gait parameters, gait asymmetry, and gait variability dependent variables, between symmetrical and asymmetrical strength groups, across standard and maximal speeds. When indicated by significant group $\times$ speed interactions, independent $T$ tests were used to assess differences between groups. Pearson product-moment correlations were utilized to investigate the relationship between kinetic (weight acceptance force and rate, push-off force and rate), spatial (step length) and temporal (step time) gait variability measures, and walking performance measures (habitual, overground speed and self-selected, maximal, treadmill speed). The significance level for all statistical tests was set at $\mathrm{P}<0.05$. 


\section{Results}

\section{Strength and Rate of Torque Development}

Strength symmetrical (SS) and strength asymmetrical (SA) groups were of similar age, height, mass, and body mass index (Table 1). The intraclass correlation coefficient for the consistency of the intralimb measurement of maximal knee extensor torque from trial 1 to trial 2 was $r_{x x}=0.96, P<0.001$. The SEM for maximal knee extensor torque was $4.09 \mathrm{Nm}$ or $0.06 \mathrm{Nm} \mathrm{kg}^{-1}$. The average intralimb difference in maximal torque from trial 1 to 2 for SA was $8.8 \pm 4.8 \%$ which was statistically lower than the average asymmetry measured between SA's limbs $(27.4 \pm 5.5 \%, \mathrm{P}<0.001)$. While no differences were observed between SS and SA groups for the maximal knee extensor torque of the strong leg $(1.55 \pm 0.29 \mathrm{Nm}$ $\mathrm{kg}^{-1}$ vs. $1.50 \pm 0.38 \mathrm{Nm} \mathrm{kg}^{-1}$ respectively, $\mathrm{P}=0.35$ ), the knee extensor torque of the weak leg was approximately $20 \%$ lower in SA $\left(1.09 \pm 0.29 \mathrm{Nm} \mathrm{kg}^{-1}\right)$ than in SS $(1.36 \pm 0.22 \mathrm{Nm}$ $\mathrm{kg}^{-1}, \mathrm{P}=0.008$, Figure 1$)$. This equated to a larger knee extensor strength asymmetry in SA $(27 \%)$ than in SS (12\%). Similarly, there were no differences in maximal RTD between SS and SA for the strong leg $\left(9.95 \pm 3.87 \mathrm{Nm} \mathrm{s}^{-1} \mathrm{~kg}^{-1}\right.$ vs. $7.79 \pm 2.79 \mathrm{Nm} \mathrm{s}^{-1} \mathrm{~kg}^{-1}$ respectively, $\mathrm{P}=0.069$ ) but the maximal $\mathrm{RTD}$ of the weak leg of SA was significantly less than SS $\left(5.11 \pm 2.12 \mathrm{Nm} \mathrm{s}^{-1} \mathrm{~kg}^{-1}\right.$ vs. $7.68 \pm 3.60 \mathrm{Nm} \mathrm{s}^{-1} \mathrm{~kg}^{-1}$ respectively, $\left.\mathrm{P}=0.025\right)$.

\section{Effect of Speed}

A significant main effect of speed was observed for several kinetic, spatial and temporal gait measures but there were no group effects or group $\times$ speed interactions for these basic gait parameters $(\mathrm{P}>0.05)$. When all subjects walked at their maximal speeds, weight acceptance force increased 15\%, push-off force 5\%, weight acceptance rate 133\%, and push-off rate $51 \%$ in comparison to walking at the standard speed (all P < 0.05 ). Furthermore, step length was $29 \%$ longer, single-limb support time $16 \%$ shorter, double-limb support time $26 \%$ shorter, and step time $19 \%$ shorter at the maximal walking speed (all P < 0.05 ). In comparison to the standard speed, gait asymmetry increased for both groups at the maximal walking speed for weight acceptance force, push-off force and single-limb support time ( $\mathrm{P}<$ 0.01 , Table 2 ). Also, gait variability increased in both groups at the maximal walking speed for weight acceptance force, push-off force, foot strike location and stride width but decreased for push-off rate and step length (Table 3).

\section{Effect of Strength Asymmetry}

Independent of speed, main effects for group existed for the asymmetry of step length, double and single-limb support times (Table 2). Paradoxically, step length asymmetry was greater in SS whereas the support time measures followed the trend for greater gait asymmetry in SA. Also, a significant main effect for group existed for the variability of single-limb support time indicating that SA had nearly 1.5 fold greater variability for this measure (Table 3).

There was a significant group $\times$ speed interaction $(P=0.017)$ for the asymmetry of weight acceptance force where SS had a $17 \%$ increase in asymmetry as speed increased from standard to maximal and SA had $185 \%$ increase at the maximal speed (Figure 2A, Table 2). A significant group $\times$ speed interaction $(P=0.020)$ also occurred for the variability of weight acceptance force. No statistical differences existed between groups at the standard speed, and although both SS and SA groups had increased weight acceptance force variability at the maximal speed ( $37 \%$ and $102 \%$ increase respectively), the change was statistically greater in SA (Figure 2B, Table 3). Figure 3 shows an example of how the vertical ground reaction force varied from step-to-step for a subject with symmetrical strength (Panel A) and one with asymmetrical strength (Panel B). Panel B demonstrates the greater variability and asymmetry during the weight acceptance phase ( $1^{\text {st }}$ force peak) 
exemplified by SA at the maximal speed. A third group $\times$ speed interaction $(\mathrm{P}=0.034)$

existed for step length variability whereby SS had a greater reduction in step length

variability as speed increased than did SA (Table 3).

\section{Association between Gait Variability Measures}

At the standard speed, significant, positive, low-strength correlations existed between the $\mathrm{CV}$ of weight acceptance peak force and the $\mathrm{CV}$ of step length $(\mathrm{r}=0.44, \mathrm{P}=0.015)$, between the CV of push-off peak force and the CV step length $(r=0.35, P=0.045)$, and between the $\mathrm{CV}$ of weight acceptance rate and the $\mathrm{CV}$ of step length $(\mathrm{r}=0.46, \mathrm{P}=0.012)$. At the maximal speed, only the $\mathrm{CV}$ of push-off rate and the $\mathrm{CV}$ of step length $(\mathrm{r}=0.60, \mathrm{P}=$ 0.001 ) demonstrated a significant, positive, moderate-strength correlation. There were no significant correlations between kinetic gait variability measures and the step time variability measure at either standard or maximal speeds.

\section{Mobility}

Habitual over-ground gait speed was $19 \%$ slower in SA $(\mathrm{P}=0.033)$ while the maximal treadmill gait speeds were similar between the two groups $(P=0.28$, Table 1$)$. The maximal speed CV for weight acceptance peak force had a low-strength, negative correlation to habitual, overground walking speed $(\mathrm{r}=-0.43, \mathrm{P}=0.018)$ but not to self-selected maximal treadmill speed $(\mathrm{r}=-0.27, \mathrm{P}=0.105)$, nor was the $\mathrm{CV}$ of weight acceptance peak force from the standard speed trial correlated to habitual, overground speed $(r=-0.13, P=0.274)$ or self-selected maximal speed $(\mathrm{r}=0.30, \mathrm{P}=0.445)$.

\section{Discussion}

In the present study we investigated the relationship between lower-extremity strength asymmetry (i.e. side-to-side differences in strength, Eq. 1), gait asymmetry (i.e. side-to-side differences in spatial, temporal and kinetic gait parameters, Eq. 1), and gait variability (i.e. inconsistency of spatial, temporal and kinetic gait parameters from step-to-step, Eq. 2) in older women. This study supports the hypothesis that older women with knee extensor strength asymmetry greater than $20 \%$ demonstrate increased gait asymmetry and variability, particularly for weight acceptance peak force, single-limb and double-limb support times. Asymmetrical strength subjects also had slower, overground walking speeds demonstrating a connection between strength asymmetry and mobility. An important finding was that for all subjects, walking at near-maximal speeds increased gait asymmetry and variability for most measures, but for weight acceptance force, the increase was greater in those with asymmetrical strength.

\section{Strength Asymmetry}

Participants in this study were separated into symmetrical and asymmetrical strength groups using a criterion of $20 \%$ difference between strong and weak legs. The standard was based on previous studies demonstrating average strength asymmetry values of $15-20 \%$ in older adults $(27,33)$. Because the comparison of gait asymmetry and variability between groups is dependent on the appropriate classification of individuals via bilateral strength asymmetry, it is imperative to establish that the measured strength asymmetry is greater than the intralimb variability of strength measurement $(12,13)$. In this study the measurement of maximal torque from trial 1 to 2 showed excellent consistency $\left(r_{x x}=0.96\right)$, the difference in knee extensor strength between strong and weak legs for SA $\left(0.41 \mathrm{Nm} \mathrm{kg}^{-1}\right)$ was much greater than the SEM $\left(0.06 \mathrm{Nm} \mathrm{kg}^{-1}\right)$, and the average strength asymmetry for SA $(27.4 \%)$ was significantly greater than the average intralimb difference in strength from trial 1 to 2 (8.8\%). It therefore appears that the bilateral strength asymmetry observed in this study is a veritable phenomenon that exceeds the variability of strength measurement. 
When the strong leg peak knee extensor torque was compared between SS and SA, no differences existed. The strong leg of both groups was at or above the $1.5 \mathrm{Nm} \mathrm{kg}^{-1}$ criterion that has been identified as a cutpoint for risk of mobility limitation $(10,22,28)$. In contrast, SA's peak knee extensor torque of the weak leg was $20 \%$ lower than SS and was well below the $1.5 \mathrm{Nm} \mathrm{kg}^{-1}$ criterion. Similarly, the weak leg knee extensor RTD was 33\% lower in SA than SS and both groups demonstrated greater asymmetry for RTD than for peak torque. With our analysis it was not possible to separately study the influence of strength and RTD asymmetry as these measures of neuromuscular performance are related to each other (36), and both have been shown to affect kinetic gait parameters (21). However, studies by both Carabello et al. and Skelton et al. suggest that asymmetry in the ability to rapidly generate force may be more closely associated with fall risk and mobility than strength asymmetry (7, $33)$.

It is likely that in individuals with asymmetrical strength and power that the weak leg may be a limiting factor in the performance of functional tasks like rising from a chair, ascending and descending stairs, and walking. In this study, the habitual, overground gait speed of SA was $19 \%$ slower than SS and was below the $1.2 \mathrm{~m} \mathrm{~s}^{-1}$ speed required to cross the street at a signaled intersection, a benchmark routinely used to classify mobility limitation (29). Also, while only $23 \%$ of SS exhibited habitual gait speeds below $1.2 \mathrm{~m} \mathrm{~s}^{-1}, 55 \%$ of SA were below this criterion measure. However, both SS and SA groups exhibited habitual gait speeds above the $1.0 \mathrm{~m} \mathrm{~s}^{-1}$ threshold shown to identify high risk of adverse health outcomes that include lower-extremity limitation, hospitalization, and death (8). While Carabello et al. demonstrated that older adults with mobility limitation had greater power asymmetry than those with normal mobility, they showed no correlation between knee extensor strength asymmetry and measures of physical function, including gait speed (7). Likewise, we found no statistical difference between groups for self-selected, maximal treadmill speed indicating that the relationship between strength asymmetry and walking speed deserves further study.

\section{Gait Asymmetry}

Much attention has been given to considering neurological control of the automaticity of gait, including discussion of the role of central pattern generators, sensory feedback, and the level of the central nervous system at which gait rhythmicity is controlled (e.g spinal and supraspinal levels) $(11,24,30)$. We do not discount the role of the nervous system in the control of symmetrical, cyclical gait, but have focused this paper on the potential gait asymmetry and variability that could result from an imbalance in supportive and propulsive forces due to strength asymmetry. The strength asymmetry, and subsequent gait asymmetry, demonstrated in this study could however be driven by central nervous and/or peripheral factors. For example, there may be alterations in the aging central nervous system that result in decreased agonist activation and/or increased antagonist coactivation or there may be peripheral alterations such as reduced muscle cross-sectional area and contractile dysfunction that contribute to asymmetry (19). Future studies that delineate the central and peripheral influences of strength asymmetry could provide useful information in preventing or reversing strength and gait asymmetries in older adults.

In the calculation of bilateral gait asymmetry, our initial intention was to determine if participants relied more on the strong leg during walking, for example greater weight acceptance forces or step length with the strong leg. However, it became apparent that although there was a relationship between strength asymmetry and gait asymmetry, there was no systematic reliance on the strong leg. For instance, 58\% of the subjects had greater weight acceptance peak force with the strong leg and $38 \%$ had longer step lengths with the strong leg. As a result, the absolute value of the weak - strong leg difference was used to define gait asymmetry (see Eq. 1). This analysis revealed that SA demonstrated greater single and double-limb support time asymmetry across the two walking speeds, and greater 
weight acceptance force asymmetry at the maximal speed. It should be noted that this study only considered the asymmetry of knee extension strength and it is plausible that strength asymmetries for other joint actions did not exhibit the same laterality (38). Furthermore, weak knee extensor strength could be compensated for within the same limb by the ankle or hip musculature, as Winter has shown (40).

Gait asymmetry has been suggested to be due to limb dominance as well as imbalanced strength of lower-extremity muscles, and it is known that gait asymmetries exist as a result of unilateral musculoskeletal injury, hemiparetic disease, and limb length inequalities (23, 30). Sadeghi et al. showed in a three-dimensional, kinetic analysis of healthy elderly gait that on average there was a 9\% difference in the total work performed between legs, and peak powers differed at the knee by as much as $36 \%$ at foot strike (31). Maupas et al. have provided one of the few studies that has investigated the relationship between lowerextremity strength and gait symmetry during walking (23). They found that neither knee flexion nor extension strength was related to knee range of motion asymmetry; however, their young, healthy subjects did not have side-to-side strength imbalances. As such, the results presented here are some of the first indications that lower-extremity strength asymmetry leads to increased gait asymmetry in older adults. These findings are important because, like gait variability, gait asymmetry has been shown to be higher in elderly fallers, possibly due to disturbance of medial-lateral balance as Yogev et al. have suggested (16, 42). Bautmans et al. provide additional support for the relationship between asymmetry and function as they demonstrated in older adults that step time asymmetry and irregularity were related to Tinetti gait (e.g. step length and width) and balance (e.g. postural stability during standing) scores, dependency in activities of daily living (e.g. dressing, bathing, rising from a chair), and fall history (4).

An important finding of the current study is that walking at near-maximal speeds increased the asymmetry of weight acceptance and push-off forces in both groups of older women. These results are in agreement with the work of Seeley and colleagues that showed in young adults that support impulse asymmetry only occurred at fast speeds (32). What is particularly interesting is that there was a group $\times$ speed interaction whereby SA had a greater increase in weight acceptance force asymmetry at the maximal speed (Fig. 2A). It is conceivable that as SA walked at near-maximal speed, and utilized a high percentage of their capacity to produce force, the deficit in strength between legs was manifested in the asymmetry of the weight acceptance forces. An alternative perspective is that to achieve faster walking speeds SA may have compensated for the weak leg by producing greater forces with the strong leg. Figure 3 demonstrates these theories by comparing a subject with symmetrical strength and gait (Fig. 3A) to one with asymmetrical strength and an asymmetrical, kinetic gait pattern (Fig. 3B). It is interesting to note that as a result of force asymmetry, the subject in 3B produced approximately $20 \%$ greater weight acceptance peak force than the subject in 3A, despite walking at nearly identical, self-selected maximal speeds. This comparison should lead researchers to question the relationships between strength asymmetry, reserve force capacity, fall risk, and the development of fatigue during prolonged walking.

\section{Gait variability}

Increasing speed from a standard speed of $0.83 \mathrm{~m} \mathrm{~s}^{-1}$ to self-selected maximal speed $(\approx 1.4$ $\mathrm{m} \mathrm{s}^{-1}$ ) altered spatial and kinetic gait variability in both groups, but did not affect the temporal measures of variability. Specifically, weight acceptance force, push-off force, foot strike location, and stride width variabilities increased at the maximal speed, and push off rate and step length variability decreased. These results agree with Jordan et al. who showed similar increases in young adults for weight acceptance and push-off force variability as speed was raised (17). A novel aspect of this study is that despite finding no group 
differences in weight acceptance force variability at the standard speed, the increase seen at maximal speed was greater for those with strength asymmetry (102\% vs. 37\%, Fig. 2B). Similarly Barak et al. showed that elderly fallers had significantly greater hip range of motion variability than non-fallers, but only at faster walking speeds (2). The relationships between kinetic gait variability, fall risk, and mobility have not been adequately studied, so the clinical significance of this finding is not yet clear. However, our preliminary analysis of the association between kinetic gait variability and function showed an inverse correlation between weight acceptance force $\mathrm{CV}$ and habitual, overground walking speed $(\mathrm{r}=-0.43)$. Because increased speed is associated with increased force variability and mediolateral spatial variability, it is plausible that SA chose slower, habitual walking speeds to maintain a rhythmical, predictable gait pattern that minimized their risk for balance perturbations and falls, as has been previously postulated $(39,41)$.

Since the relationship between strength asymmetry and increased weight acceptance force variability is a new finding, the results should be replicated, and an effort should be made to provide an explanation for the association. We speculate that a number of neurological, muscular, and methodological factors could have elicited these findings. First, because gait variability measures were computed from step to step across both legs, the asymmetry of gait identified in SA likely contributed to the overall variability observed (12). Second, it is possible that at submaximal speeds SA was able to match the force of opposing legs through differential nervous activation. However, as they approached maximal speed each leg would be operating closer to its maximal force capacity resulting in side-to-side differences in the supportive forces applied to the ground. In addition, as a muscle gets closer to its maximal force capacity the absolute variability (SD) of its force output increases (37). Hence, if opposing legs have different maximal force capacities, the variability of force output may change independently for each leg as a product of walking speed, contributing to an increase in kinetic variability at faster speeds. Similarly, if as a result of differing force capacities, the muscles of opposing legs fatigue at different rates, side-to-side and within leg force variability may be introduced by the fatigued muscle (9). Ultimately, the variability of the supportive and propulsive forces generated during walking may contribute to the welldocumented greater variability of spatial and temporal gait measures seen in older individuals $(6,14)$. The positive correlations between kinetic variability and step length variability seen in this study support this idea.

As speed increased, temporal and anteroposterior spatial measures of gait variability were preserved despite increased kinetic and mediolateral variability. Contrary to expectations, step length asymmetry and variability were higher in SS at the standard speed. It is possible that this occurred because the standard speed $\left(0.83 \mathrm{~m} \mathrm{~s}^{-1}\right)$ was considerably below preferred speed for this group $\left(\mathrm{SS}=1.29 \mathrm{~m} \mathrm{~s}^{-1}, \mathrm{SA}=1.05 \mathrm{~m} \mathrm{~s}^{-1}\right.$ ) and increasing speed allowed them to adopt a more customary gait pattern that reduced asymmetry and variability. These findings are in agreement with Kang and Dingwell who showed that step length variability tended to decrease as older individuals increased from slow to preferred walking speed (18) and by Jordan et al. who showed in younger adults that step length variability decreased with increased speed (17). However, across both speeds, SA had greater variability for single-limb support time and a statistical trend for greater double-limb support time variability $(\mathrm{P}=0.06)$. It is possible that because of the greater kinetic variability observed in SA, they altered support and swing times with each step to maintain the consistency of step time and length which were shown not to differ between groups.

A limitation of the current study is that gait assessment was performed during treadmill walking as opposed to overground walking. While this limits external validity of the results to some extent, treadmill walking has been shown to be biomechanically similar to overground walking (25). It is however possible that walking on a treadmill influenced the 
walking pattern and variability by decreasing stride length, increasing cadence, and increasing stride width, the latter indicating a challenge to balance (34). Nevertheless, an instrumented treadmill is well suited to studying gait variability and asymmetry over repeated footstrikes, particularly for kinetic measures which usually require subjects to target force plates when walking across a room or to wear pressure sensitive insole systems which have been shown to be less sensitive to ground reaction forces (3). This study classified SS and SA subjects utilizing torque from an isometric knee extension test. While it is possible that strength asymmetries could be calculated from dynamic contractions, the methods employed in the study allowed us to identify individuals with strength values above or below the risk for mobility limitations based on previous studies $(10,22,28)$. In addition, the strength testing was performed before the walking trials which may have influenced participants' neuromuscular performance during the gait testing. It is also important to note that the subjects in this study were independent and healthy, thus limiting the generalizability of the results.

Strength asymmetry, gait asymmetry, and gait variability have each been independently shown to be related to falling and mobility status in older adults, but this study demonstrates that these variables are likely interrelated. Older women with knee extensor strength asymmetry greater than $20 \%$ had greater weight acceptance force, double and single-limb support time asymmetry, and greater weight acceptance force and single-limb support time variability than their strength-symmetrical peers. Strength asymmetry was associated with slower, habitual, overground walking speed but did not affect basic gait parameters at either standard or maximal speeds. Independent of the level of strength asymmetry, increasing speed was associated with greater force asymmetry and variability, and greater mediolateral spatial variability, but lower stride length and push-off rate variability. With respect to these findings, it is our recommendation that the clinical assessment of strength in older adults be performed unilaterally with the intention of documenting strength asymmetry to potentially identify individuals at risk of falls or mobility limitations. Resistance exercise programs should be designed to restore strength symmetry, possibly through unilateral exercise targeting the weak limb. Although we have shown a connection between strength asymmetry and altered gait, this study did not specifically assess the relationships between strength asymmetry, fall risk, and functional mobility. Therefore, future research should investigate these relationships and prospectively determine if restoration of strength symmetry results in improved mobility, and reduced gait asymmetry, gait variability, and risk of falling.

\section{Acknowledgments}

D.P. LaRoche was supported by the National Institute on Aging via NIH Grant L30 AG038028-01.

\section{References}

1. AGS. Guideline for the prevention of falls in older persons. American Geriatrics Society, British Geriatrics Society, and American Academy of Orthopaedic Surgeons Panel on Falls Prevention. J Am Geriatr Soc. 2001; 49(5):664-72. [PubMed: 11380764]

2. Barak Y, Wagenaar RC, Holt KG. Gait characteristics of elderly people with a history of falls: a dynamic approach. Physical therapy. 2006; 86(11):1501-10. [PubMed: 17079750]

3. Barnett S, Cunningham JL, West S. A comparison of vertical force and temporal parameters produced by an in-shoe pressure measuring system and a force platform. Clinical biomechanics. 2000; 15(10):781-5. [PubMed: 11050363]

4. Bautmans I, Jansen B, Van Keymolen B, Mets T. Reliability and clinical correlates of 3Daccelerometry based gait analysis outcomes according to age and fall-risk. Gait \& posture. 2011; 33(3):366-72. [PubMed: 21227697] 
5. Brach JS, Berlin JE, VanSwearingen JM, Newman AB, Studenski SA. Too much or too little step width variability is associated with a fall history in older persons who walk at or near normal gait speed. J Neuroeng Rehabil. 2005; 2:21. [PubMed: 16042812]

6. Brach JS, Berthold R, Craik R, VanSwearingen JM, Newman AB. Gait variability in communitydwelling older adults. Journal of the American Geriatrics Society. 2001; 49(12):1646-50. [PubMed: 11843998]

7. Carabello RJ, Reid KF, Clark DJ, Phillips EM, Fielding RA. Lower extremity strength and power asymmetry assessment in healthy and mobility-limited populations: reliability and association with physical functioning. Aging clinical and experimental research. 2010; 22(4):324-9. [PubMed: 19940556]

8. Cesari M, Kritchevsky SB, Penninx BW, Nicklas BJ, Simonsick EM, Newman AB, Tylavsky FA, Brach JS, Satterfield S, Bauer DC, Visser M, Rubin SM, Harris TB, Pahor M. Prognostic value of usual gait speed in well-functioning older people--results from the Health, Aging and Body Composition Study. Journal of the American Geriatrics Society. 2005; 53(10):1675-80. [PubMed: 16181165]

9. Contessa P, Adam A, De Luca CJ. Motor unit control and force fluctuation during fatigue. Journal of applied physiology. 2009; 107(1):235-43. [PubMed: 19390005]

10. Cress ME, Meyer M. Maximal voluntary and functional performance levels needed for independence in adults aged 65 to 97 years. Phys Ther. 2003; 83(1):37-48. [PubMed: 12495411]

11. Duysens J, Van de Crommert HW. Neural control of locomotion; The central pattern generator from cats to humans. Gait \& posture. 1998; 7(2):131-41. [PubMed: 10200383]

12. Exell TA, Irwin G, Gittoes MJ, Kerwin DG. Implications of intra-limb variability on asymmetry analyses. J Sports Sci. 2012; 30(4):403-9. [PubMed: 22248309]

13. Giakas G, Baltzopoulos V. Time and frequency domain analysis of ground reaction forces during walking: An investigation of variability and symmetry. Gait Posture. 1997; 5:189-97.

14. Hausdorff JM, Edelberg HK, Mitchell SL, Goldberger AL, Wei JY. Increased gait unsteadiness in community-dwelling elderly fallers. Archives of physical medicine and rehabilitation. 1997; 78(3): 278-83. [PubMed: 9084350]

15. Hausdorff JM, Rios DA, Edelberg HK. Gait variability and fall risk in community-living older adults: a 1-year prospective study. Arch Phys Med Rehabil. 2001; 82(8):1050-6. [PubMed: 11494184]

16. Hill K, Schwarz J, Flicker L, Carroll S. Falls among healthy, community-dwelling, older women: a prospective study of frequency, circumstances, consequences and prediction accuracy. Aust N Z J Public Health. 1999; 23(1):41-8. [PubMed: 10083688]

17. Jordan K, Challis JH, Newell KM. Walking speed influences on gait cycle variability. Gait \& posture. 2007; 26(1):128-34. [PubMed: 16982195]

18. Kang HG, Dingwell JB. Separating the effects of age and walking speed on gait variability. Gait \& posture. 2008; 27(4):572-7. [PubMed: 17768055]

19. Klass M, Baudry S, Duchateau J. Voluntary activation during maximal contraction with advancing age: a brief review. European journal of applied physiology. 2007; 100(5):543-51. [PubMed: 16763836]

20. Lanshammar K, Ribom EL. Differences in muscle strength in dominant and non-dominant leg in females aged 20-39 years--a population-based study. Phys Ther Sport. 2011; 12(2):76-9. [PubMed: 21496769]

21. LaRoche DP, Millett ED, Kralian RJ. Low strength is related to diminished ground reaction forces and walking performance in older women. Gait \& posture. 2011; 33(4):668-72. [PubMed: 21458271]

22. Manini TM, Visser M, Won-Park S, Patel KV, Strotmeyer ES, Chen H, Goodpaster B, De Rekeneire N, Newman AB, Simonsick EM, Kritchevsky SB, Ryder K, Schwartz AV, Harris TB. Knee extension strength cutpoints for maintaining mobility. J Am Geriatr Soc. 2007; 55(3):451-7. [PubMed: 17341251]

23. Maupas E, Paysant J, Datie AM, Martinet N, Andre JM. Functional asymmetries of the lower limbs. A comparison between clinical assessment of laterality, isokinetic evaluation and 
electrogoniometric monitoring of knees during walking. Gait \& posture. 2002; 16(3):304-12. [PubMed: 12443956]

24. Nielsen JB. How we walk: central control of muscle activity during human walking. Neuroscientist. 2003; 9(3):195-204. [PubMed: 15065815]

25. Parvataneni K, Ploeg L, Olney SJ, Brouwer B. Kinematic, kinetic and metabolic parameters of treadmill versus overground walking in healthy older adults. Clinical biomechanics. 2009; 24(1): 95-100. [PubMed: 18976839]

26. Paterson K, Hill K, Lythgo N. Stride dynamics, gait variability and prospective falls risk in active community dwelling older women. Gait \& posture. 2011; 33(2):251-5. [PubMed: 21167715]

27. Perry MC, Carville SF, Smith IC, Rutherford OM, Newham DJ. Strength, power output and symmetry of leg muscles: effect of age and history of falling. Eur J Appl Physiol. 2007; 100(5): 553-61. [PubMed: 16847676]

28. Ploutz-Snyder LL, Manini T, Ploutz-Snyder RJ, Wolf DA. Functionally relevant thresholds of quadriceps femoris strength. J Gerontol A Biol Sci Med Sci. 2002; 57(4):B144-52. [PubMed: 11909879]

29. Rantanen T, Guralnik JM, Izmirlian G, Williamson JD, Simonsick EM, Ferrucci L, Fried LP. Association of muscle strength with maximum walking speed in disabled older women. Am J Phys Med Rehabil. 1998; 77(4):299-305. [PubMed: 9715919]

30. Sadeghi H, Allard P, Prince F, Labelle H. Symmetry and limb dominance in able-bodied gait: a review. Gait \& posture. 2000; 12(1):34-45. [PubMed: 10996295]

31. Sadeghi H, Prince F, Zabjek KF, Labelle H. Simultaneous, bilateral, and three-dimensional gait analysis of elderly people without impairments. American journal of physical medicine \& rehabilitation / Association of Academic Physiatrists. 2004; 83(2):112-23.

32. Seeley MK, Umberger BR, Shapiro R. A test of the functional asymmetry hypothesis in walking. Gait \& posture. 2008; 28(1):24-8. [PubMed: 17997095]

33. Skelton DA, Kennedy J, Rutherford OM. Explosive power and asymmetry in leg muscle function in frequent fallers and non-fallers aged over 65. Age Ageing. 2002; 31(2):119-25. [PubMed: 11937474]

34. Stolze H, Kuhtz-Buschbeck JP, Mondwurf C, Boczek-Funcke A, Johnk K, Deuschl G, Illert M. Gait analysis during treadmill and overground locomotion in children and adults. Electroencephalogr Clin Neurophysiol. 1997; 105(6):490-7. [PubMed: 9448652]

35. Suetta C, Aagaard P, Magnusson SP, Andersen LL, Sipila S, Rosted A, Jakobsen AK, Duus B, Kjaer M. Muscle size, neuromuscular activation, and rapid force characteristics in elderly men and women: effects of unilateral long-term disuse due to hip-osteoarthritis. Journal of applied physiology. 2007; 102(3):942-8. [PubMed: 17122381]

36. Thelen DG, Schultz AB, Alexander NB, Ashton-Miller JA. Effects of age on rapid ankle torque development. J Gerontol A Biol Sci Med Sci. 1996; 51(5):M226-32. [PubMed: 8808994]

37. Tracy BL. Force control is impaired in the ankle plantarflexors of elderly adults. European journal of applied physiology. 2007; 101(5):629-36. [PubMed: 17701201]

38. Vagenas G, Hoshizaki B. Functional Asymmetries and Lateral Dominance in the Lower Limbs of Distance Runners. Internationl journal of sport biomechanics. 1991; 7:311-29.

39. Wall JC, Hogan DB, Turnbull GI, Fox RA. The kinematics of idiopathic gait disorder. A comparison with healthy young and elderly females. Scandinavian journal of rehabilitation medicine. 1991; 23(3):159-64. [PubMed: 1962159]

40. Winter DA. Overall principle of lower limb support during stance phase of gait. J Biomech. 1980; 13(11):923-7. [PubMed: 7275999]

41. Winter DA, Patla AE, Frank JS, Walt SE. Biomechanical walking pattern changes in the fit and healthy elderly. Physical therapy. 1990; 70(6):340-7. [PubMed: 2345777]

42. Yogev G, Plotnik M, Peretz C, Giladi N, Hausdorff JM. Gait asymmetry in patients with Parkinson's disease and elderly fallers: when does the bilateral coordination of gait require attention? Experimental brain research Experimentelle Hirnforschung Experimentation cerebrale. 2007; 177(3):336-46. [PubMed: 16972073] 
A.

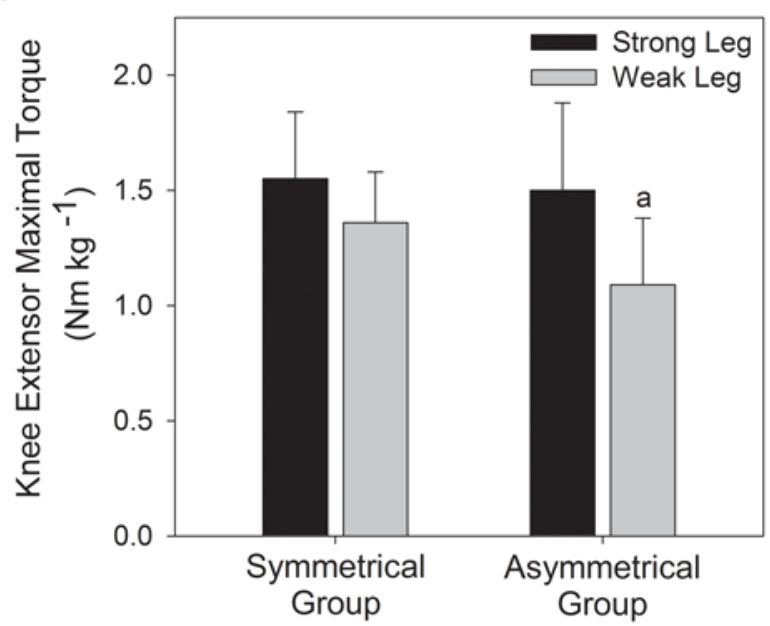

B.

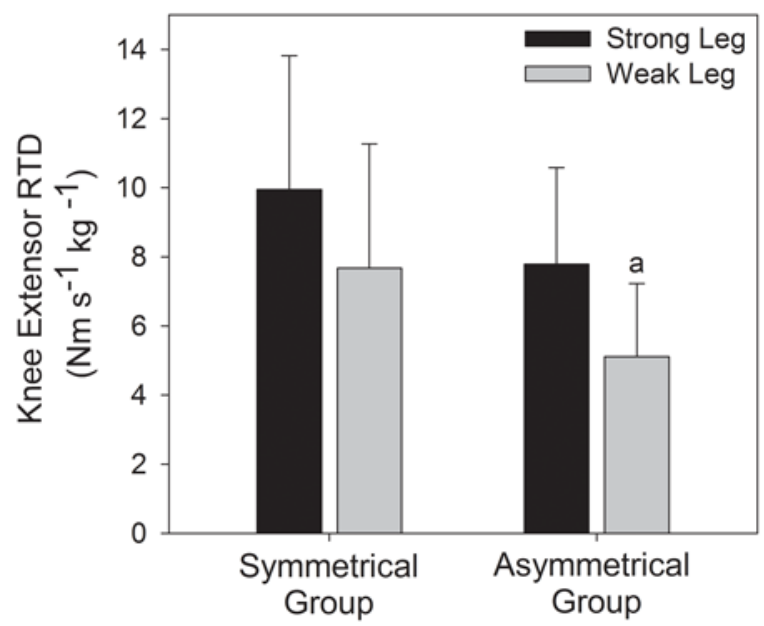

Figure 1.

Comparison of knee extensor strength (A) and rate of torque development (RTD) (B) between the strong and weak legs of those with symmetrical and asymmetrical strength. $\mathrm{a}=$ significant difference between groups for the weak leg $\mathrm{P}<0.05$ 
A.

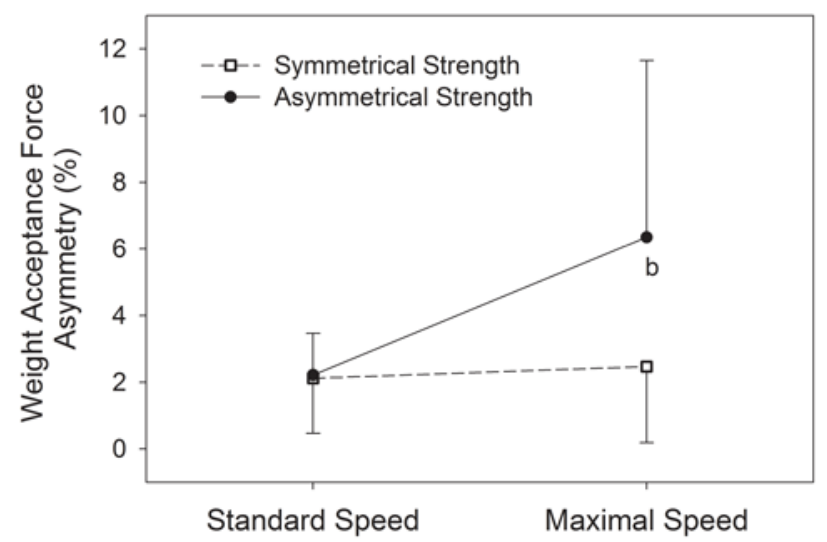

B.

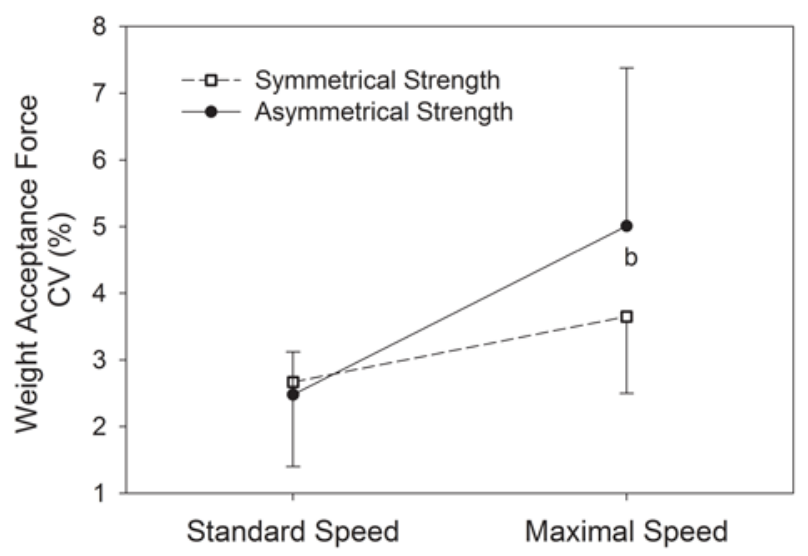

Figure 2.

Asymmetry (A) and coefficient of variation (CV) (B) of weight acceptance peak force compared between those with symmetrical and asymmetrical knee extensor strength at standard and maximal speeds.

$\mathrm{b}=$ significant group $\times$ speed interaction, $\mathrm{P}<0.05$ 
A.

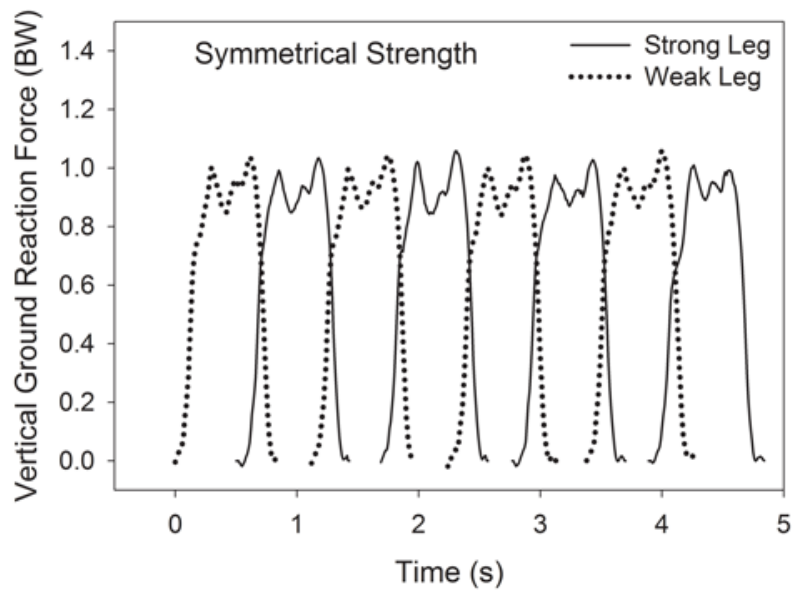

B.

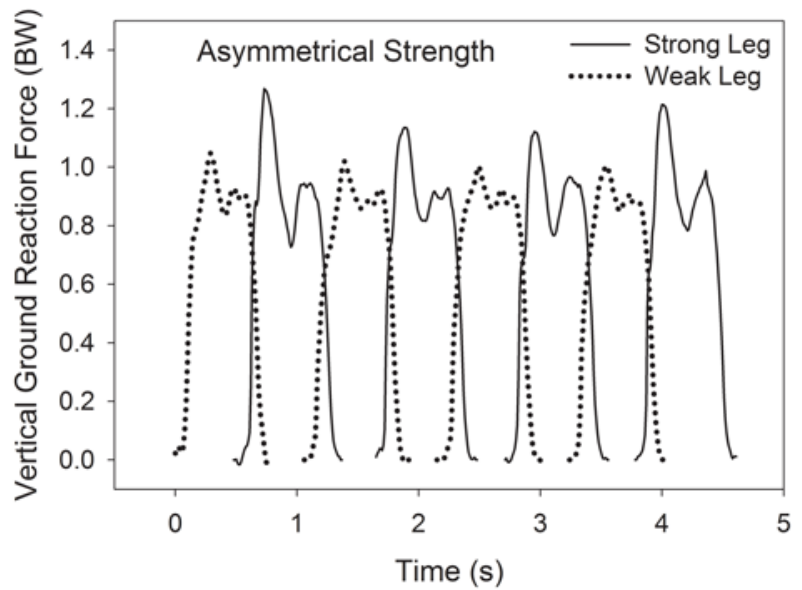

Figure 3.

Representative vertical ground reaction force data of a subject with symmetrical strength with an invariable, symmetrical gait pattern (A) and a subject who had asymmetrical strength with a variable, asymmetrical gait pattern (B) while walking at similar maximal speeds $\left(\approx 1.03 \mathrm{~m} \mathrm{~s}^{-1}\right)$.

$\mathrm{BW}=$ body weights 
Table 1

\section{Subject Characteristics}

\begin{tabular}{lccc}
\hline & Symmetrical Strength & Asymmetrical Strength & P - values \\
\hline Age (yr) & $70.9 \pm 4.7$ & $72.5 \pm 4.6$ & 0.192 \\
& $(66.0-81.0)$ & $(65-77)$ & \\
Height (m) & $1.61 \pm 0.05$ & $1.60 \pm 0.07$ & 0.277 \\
Mass (kg) & $(1.53-1.68)$ & $(1.52-1.73)$ & \\
& $63.6 \pm 7.7$ & $66.5 \pm 9.9$ & 0.208 \\
BMI (kg m & $(54.0-77.5)$ & $(49.4-86.1)$ & \\
& $24.5 \pm 3.3$ & $26.2 \pm 4.3$ & 0.142 \\
Strength Asymmetry $(\%)$ & $(19.7-29.8)$ & $(17.8-33.3)$ & \\
& $11.7 \pm 5.4$ & $27.4 \pm 5.5$ & $<0.001$ \\
RTD Asymmetry $(\%)$ & $(1.7-19.2)$ & $(22.2-38.2)$ & \\
& $23.4 \pm 17.1$ & $33.2 \pm 18.2$ & 0.095 \\
Habitual Gait Speed $\left(\mathrm{m} \mathrm{s}^{-1}\right)$ & $(1.3-48.9)$ & $(0.1-60.6)$ & \\
& $1.29 \pm 0.16$ & $1.05 \pm 0.23$ & 0.033 \\
Maximal Gait Speed $\left(\mathrm{m} \mathrm{s}^{-1}\right)$ & $(1.05-1.60)$ & $(0.85-1.90)$ & \\
& $1.43 \pm 0.26$ & $1.36 \pm 0.24$ & 0.280 \\
\hline
\end{tabular}

All values are mean \pm standard deviation (range)

BMI $=$ Body Mass Index

$\mathrm{RTD}=$ Rate of torque development 


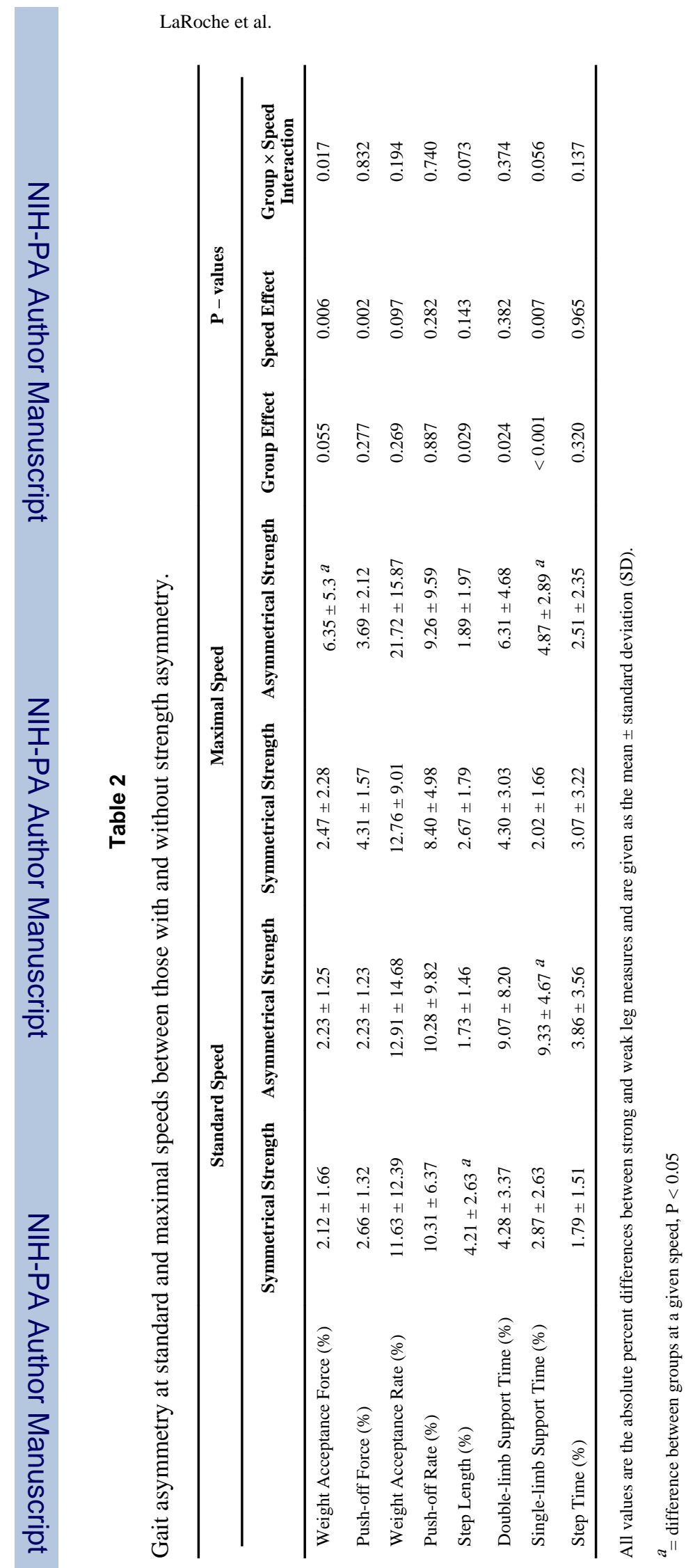

Page 18 


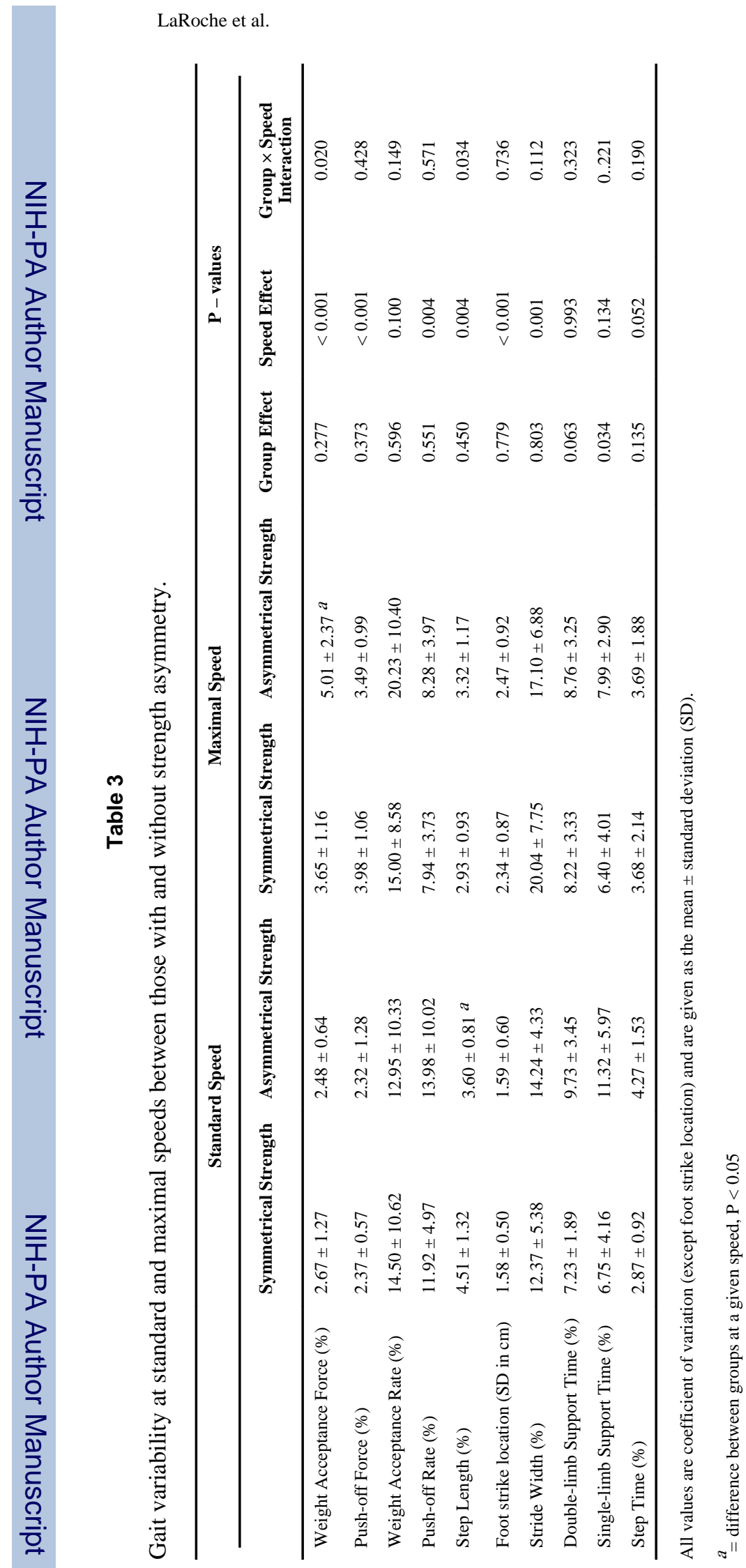

Page 19 